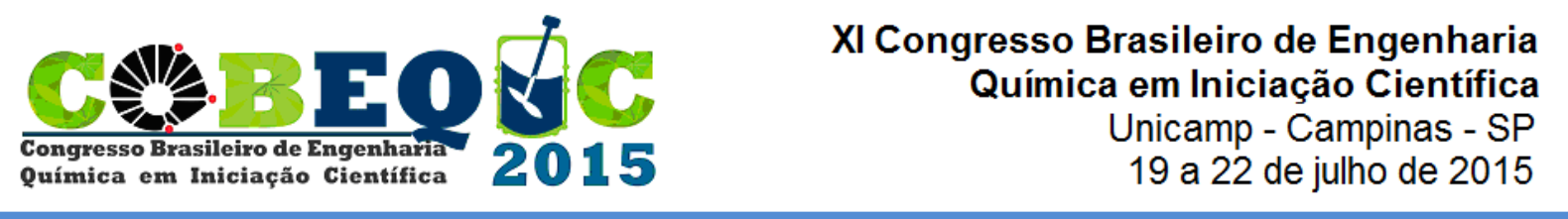

\title{
USO DE PLANEJAMENTO ESTATÍSTICO DE EXPERIMENTOS NA DETERMINAÇÃO DO PH E TEMPERATUTA ÓTIMOS DE $\alpha$-AMILASE DE BACILLUS LICHENIFORMIS
}

\author{
A. TRENTIN ${ }^{1}$, A. L. ASTOLFI ${ }^{1}$, J. ZENI ${ }^{1}$, A. C. SCHUMANN ${ }^{1}$, C. M. BOCALON ${ }^{1}$, J. \\ COLLA $^{1}$, N. L. D. NYARI ${ }^{1}$ \\ ${ }^{1}$ Universidade Regional Integrada do Alto Uruguai e das Missões, Departamento de \\ Engenharia de Alimentos \\ E-mail para contato: jamilezeni@uricer.edu.br
}

\begin{abstract}
RESUMO - Neste trabalho objetivou-se caracterizar parcialmente a $\alpha$-amilase de Bacillus licheniformis em termo de $\mathrm{pH}$ e temperatura ótimos usando planejamento estatístico de experimentos. Para a determinação dos valores ótimos de temperatura e $\mathrm{pH}$ foi realizado um planejamento fatorial completo $2^{2}$, onde o pH ira variar de 5 a 11 e temperatura de 30 a $80^{\circ} \mathrm{C}$. A determinação da atividade enzimática da $\alpha$-amilase foi realizada adicionando-se $0,1 \mathrm{~mL}$ de enzima a $0,9 \mathrm{~mL}$ de solução de glicina $50 \mathrm{mM}$ contendo $1 \%$ de amido e incubando a reação por 10 minutos, segundo metodologia descrita por San et al. (2011). De acordo com os resultados obtidos usando o planejamento estatístico experimental, a enzima $\alpha$ - amilase apresentou a maior atividade enzimática $(8,7 \mathrm{U} / \mathrm{mL})$, com temperatura acima de $60^{\circ} \mathrm{C}$ e $\mathrm{pH}$ na faixa de 9 a 11.
\end{abstract}

\section{INTRODUÇÃO}

As enzimas são substâncias orgânicas poliméricas formadas por unidades de aminoácidos que atuam no metabolismo dos seres vivos como catalisadores naturais. Estes biocatalisadores, na maioria das vezes de estrutura proteica do tipo globular terciária ou quaternária, aceleram bastante a velocidade de uma reação termodinamicamente favorável, diminuindo a energia de ativação e estabilizando o estado de transição das reações (Nelson; Cox, 2011). As enzimas estão presentes em todos os organismos vivos sejam plantas ou animais, dos mais simples, como as formas de vida unicelulares, aos mais complexos (Spier, 2005).

A denominação das enzimas geralmente é dada de acordo com o substrato em que atuam. Assim, o termo amilase indica a ação enzimática sobre o amido (amilo), que é constituído por dois tipos de polissacarídeos: a amilose (15-20\%) e amilopectina (80-85\%) (Vieira-Júnior, 2006).

As amilases são enzimas que catalisam a hidrólise de ligações $\alpha-1-4$ glicosídicas de polissacarídeos, como glicogênio, amido ou seus produtos de degradação. Sobre o amido, atuam liberando diversos produtos, incluindo dextrinas e progressivamente pequenos polímeros compostos de unidades de glicose (Vieira-Júnior, 2006). Produzida na saliva e no pâncreas, a amilase também é produzida por diversos fungos, bactérias e vegetais (Sankalia et al., 2007). 
As enzimas sofrem os mesmos efeitos estruturais observados com as proteínas pela variação de $\mathrm{pH}$ e de temperatura, estes fatores são capazes de alterar a atividade das enzimas e, consequentemente, a velocidade das reações por elas catalisadas. Sendo assim, cada enzima possui um $\mathrm{pH}$ ótimo de atividade (onde sua atividade é máxima); para a maioria das enzimas ele está entre 4,5 e 8,0. Levando em conta que mudanças extremas de $\mathrm{pH}$ podem alterar a estrutura da enzima devido a uma repulsão de cargas. No entanto, as mudanças de $\mathrm{pH}$ que não afetam totalmente a estrutura de uma enzima, podendo apenas diminuir sua atividade por estar afetando somente resíduos do sítio catalítico (Silva et al., 2008).

Neste sentido, este trabalho teve como objetivo caracterizar parcialmente a $\alpha$-amilase de Bacillus licheniformis em termo de $\mathrm{pH}$ e temperatura ótimos usando planejamento estatístico de experimentos.

\section{METODOLOGIA}

\subsection{Enzima}

A enzima utilizada neste estudo foi a $\alpha$-amilase comercial de Bacillus licheniformis (Sigma).

\subsection{Caracterização parcial da $\alpha$-amilase}

A caracterização das enzimas é de suma importância para o estabelecimento das condições de aplicação, como temperatura e pH ótimos, uma vez que tais condições podem ter uma grande variação de acordo com a origem e os meios de produção utilizados.

Para a determinação dos valores ótimos de temperatura e $\mathrm{pH}$ foi realizado um planejamento fatorial completo $2^{2}$, onde o $\mathrm{pH}$ ira variar de 5 a 11 e temperatura de 30 a $80^{\circ} \mathrm{C}$ (Tabela 1).

Tabela 1 - Variáveis e níveis utilizados no planejamento fatorial completo $2^{2}$ de temperatura e $\mathrm{pH}$ ótimos para a $\alpha$-amilase.

\begin{tabular}{lllllll}
\hline \multirow{2}{*}{ Variáveis Independentes* } & \multirow{2}{*}{ Códigos } & \multicolumn{7}{c}{ Níveis } \\
\cline { 3 - 7 } & $\mathrm{X}_{7}$ & 5,0 & 5,54 & 7,0 & $\mathbf{+ 1}$ & $\mathbf{1 , 4 1}$ \\
\hline $\mathrm{pH}$ & $\mathrm{X}_{8}$ & 30 & 37 & 55 & 73 & 11 \\
Temperatura $\left({ }^{\circ} \mathrm{C}\right)$ & & & &
\end{tabular}

\subsection{Determinação da atividade enzimática}

A quantificação de atividade enzimática da $\alpha$-amilase foi realizada segundo metodologia descrita por San et al., (2011), onde $0,1 \mathrm{~mL}$ de enzima era adicionado a $0,9 \mathrm{~mL}$ de solução de substrato, que contem $1 \%$ solúvel amido em $50 \mathrm{mM}$ glycine- $\mathrm{NaOH}$ (pH 9,0), e a mistura então era incubada a $50{ }^{\circ} \mathrm{C}$ por 10 minutos. A quantidade de açúcares redutores produzidos foi determinada pelo método do ácido dinitrossalicilico (DNS) (Miller, 1959) com glucose como um padrão.Uma unidade de atividade da amilase foi definida como a libertação de $1 \mathrm{~mol}$ de açúcar redutor por 10 minutos a $50^{\circ} \mathrm{C}$ e $\mathrm{pH} 9$. 


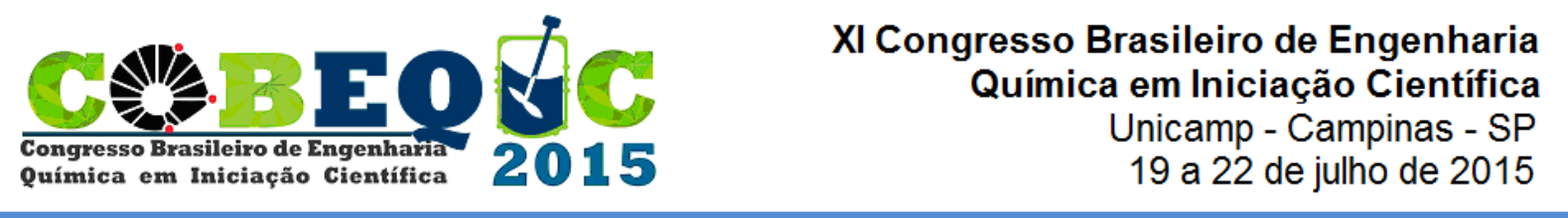

\section{RESULTADOS}

\subsection{Caracterização parcial da $\alpha$-amilase em termos de $\mathrm{pH}$ e temperatura ótimos}

A Tabela 2 apresenta a matriz do planejamento do fatorial completo $2^{2}$ com os valores codificados (reais) das variáveis independentes estudadas e as respostas em atividade de amilase.

De acordo com os resultados apresentados na Tabela 2, podemos observar que a maior atividade de amilase foi de $8,7 \mathrm{U} / \mathrm{mL}$ no ensaio 4, com temperatura de $60^{\circ} \mathrm{C}$ e $\mathrm{pH}$ de 11 .

Spier (2005) estudando $\mathrm{pH}$ e temperatura ótimos de atividade para amilase fúngica obtiveram $\mathrm{pH}$ ótimo em 6,0 e temperatura ótima entre $45-55^{\circ} \mathrm{C}$, resultado este que diferem dos encontrados no presente estudo.

Figueira et al. (2000) produziram amilases dos fungos Fusarium moniliforme e Aspergillus flavus obtendo valores de $\mathrm{pH}$ ótimos de 6,7 e 5,5 respectivamente, demonstrando a tendência de produção de amilases levemente ácidas por fungos. ótimo 8,0 .

Rasiah; Rehm (2009) obtiveram uma amilase em E. coli recombinante que possuía $\mathrm{pH}$

Tabela 2- Matriz do planejamento do fatorial completo $2^{2}$ em termos de temperatura e $\mathrm{pH}$ ótimos da enzima amilase $(\mathrm{U} / \mathrm{mL})$.

\begin{tabular}{cccc}
\hline Ensaios & $\boldsymbol{X}_{\boldsymbol{I}}$ & $\boldsymbol{X}_{\mathbf{2}}$ & Atividade $(\boldsymbol{U} / \boldsymbol{m L})$ \\
\hline 1 & $-1(5,54)$ & $-1(37)$ & 0,0 \\
2 & $1(9,8)$ & $-1(37)$ & 0,0 \\
3 & $-1(5,54)$ & $1(73)$ & 3,9 \\
4 & $1(9,8)$ & $1(73)$ & 8,7 \\
5 & $-1,41(5)$ & $0(50)$ & 0,6 \\
6 & $1,41(11)$ & $0(50)$ & 1,9 \\
7 & $0(7)$ & $-1,41(30)$ & 0,0 \\
8 & $0(7)$ & $1,41(80)$ & 0,2 \\
9 & $0(7)$ & $0(50)$ & 2,1 \\
10 & $0(7)$ & $0(50)$ & 2,6 \\
11 & $0(7)$ & $0(50)$ & 2,3 \\
\hline
\end{tabular}

*Variáveis independentes: $\mathrm{X}_{1}=\mathrm{pH}$ e $\mathrm{X}_{2}=$ Temperatura $\left({ }^{\circ} \mathrm{C}\right)$.

A Tabela 3 apresenta os coeficientes de regressão e erro padrão, valores de $\mathrm{p}$ e $\mathrm{t}$ do planejamento fatorial completo $2^{2}$ para atividade de amilase, na qual podemos observar que as variáveis Temperatura $\left({ }^{\circ} \mathrm{C}\right)(\mathrm{Q})$ e a interação entre $\mathrm{o} \mathrm{pH}$ e temperatura $(1 \mathrm{~L}$ by $2 \mathrm{~L})$ não apresentaram efeito significativo a nível de $95 \%$ na atividade de amilase $(\mathrm{U} / \mathrm{mL})$.

Tabela 3- Coeficientes de regressão e erro padrão, valores de p e t do planejamento fatorial completo $2^{2}$ para atividade de amilase. 


\begin{tabular}{lllll}
\hline & Regressão & Padrão & & \\
\hline Média & 14,289 & 0,3492 & 40,908 & 0,0005 \\
$(1)$ pH $(\mathrm{L})$ & 0,772 & 0,2142 & 3,605 & 0,0690 \\
pH $(\mathrm{Q})$ & $-1,961$ & 0,2556 & $-7,671$ & 0,0165 \\
$(2)$ Temperatura $\left({ }^{\circ} \mathrm{C}\right)(\mathrm{L})$ & 3,845 & 0,2142 & 17,949 & 0,0030 \\
Temperatura $\left({ }^{\circ} \mathrm{C}\right)(\mathrm{Q})$ & $-0,142$ & 0,2556 & $-0,558$ & 0,6327 \\
1L by 2L & 0,515 & 0,3025 & 1,702 & 0,2307 \\
\hline
\end{tabular}

*Fatores estatisticamente significativos $(\mathrm{p}<0,05)$

A Tabela 4 apresenta a analise de variância realizada a partir dos dados obtidos no planejamento fatorial completo $2^{2}$ para a atividade da $\alpha$-amilase $(\mathrm{U} / \mathrm{mL})$, onde podemos observar que o $\mathrm{F}$ calculado foi 1,33 vezes maior que o valor do $\mathrm{F}$ tabelado com um coeficiente de correlação de 0,91 , permitindo desta forma a validação do modelo (Eq. 1) e a construção de superfícies de resposta e curvas de contorno apresentadas na Figura 1 demonstrando que a máxima atividade de amilase se dará na faixa de $\mathrm{pH}$ de 7 a 9,8 e temperatura acima de $60^{\circ} \mathrm{C}$.

Tabela 4- Análise de variância para a atividade da $\alpha$-amilase $(\mathrm{U} / \mathrm{mL})$ do planejamento fatorial completo $2^{2}$.

\begin{tabular}{lllll}
\hline $\begin{array}{l}\text { Fontes de } \\
\text { Variação }\end{array}$ & $\begin{array}{l}\text { Graus de } \\
\text { Liberdade }\end{array}$ & $\begin{array}{l}\text { Soma dos } \\
\text { Quadrados }\end{array}$ & $\begin{array}{l}\text { Quadrados } \\
\text { Médios }\end{array}$ & $\begin{array}{l}\text { F } \\
\text { Calculado }\end{array}$ \\
\hline Regressão & 146,42 & 5 & 29,28 & 5,44 \\
Resíduo & 26,92 & 5 & 5,38 & \\
Falta de Ajuste & 26,1870 & 3 & & \\
Erro Puro & 0,7321 & 2 & & \\
Total & 173,3377 & 10 & &
\end{tabular}

A Equação 1 apresenta o modelo codificado de segunda ordem que descreve a atividade da amilase em função do pH e temperatura, dentro das faixas estudadas.

Amilase $=14,29-1,96 \cdot \mathrm{X}_{1}{ }^{2}+3,84 \cdot \mathrm{X}_{2}$

Onde:

Amilase $=$ Atividade de amilase $(\mathrm{U} / \mathrm{mL}) ; \mathrm{X}_{1}=\mathrm{pH}$ e $\mathrm{X}_{2}=$ Temperatura $\left({ }^{\circ} \mathrm{C}\right)$ 


\section{CEBEOTC \\ XI Congresso Brasileiro de Engenharia \\ Química em Iniciação Científica Unicamp - Campinas - SP 19 a 22 de julho de 2015}

Figura 1- Superfície de resposta e curva de contorno do $\mathrm{pH}$ e da temperatura para a atividade da amilase $(\mathrm{U} / \mathrm{mL})$.
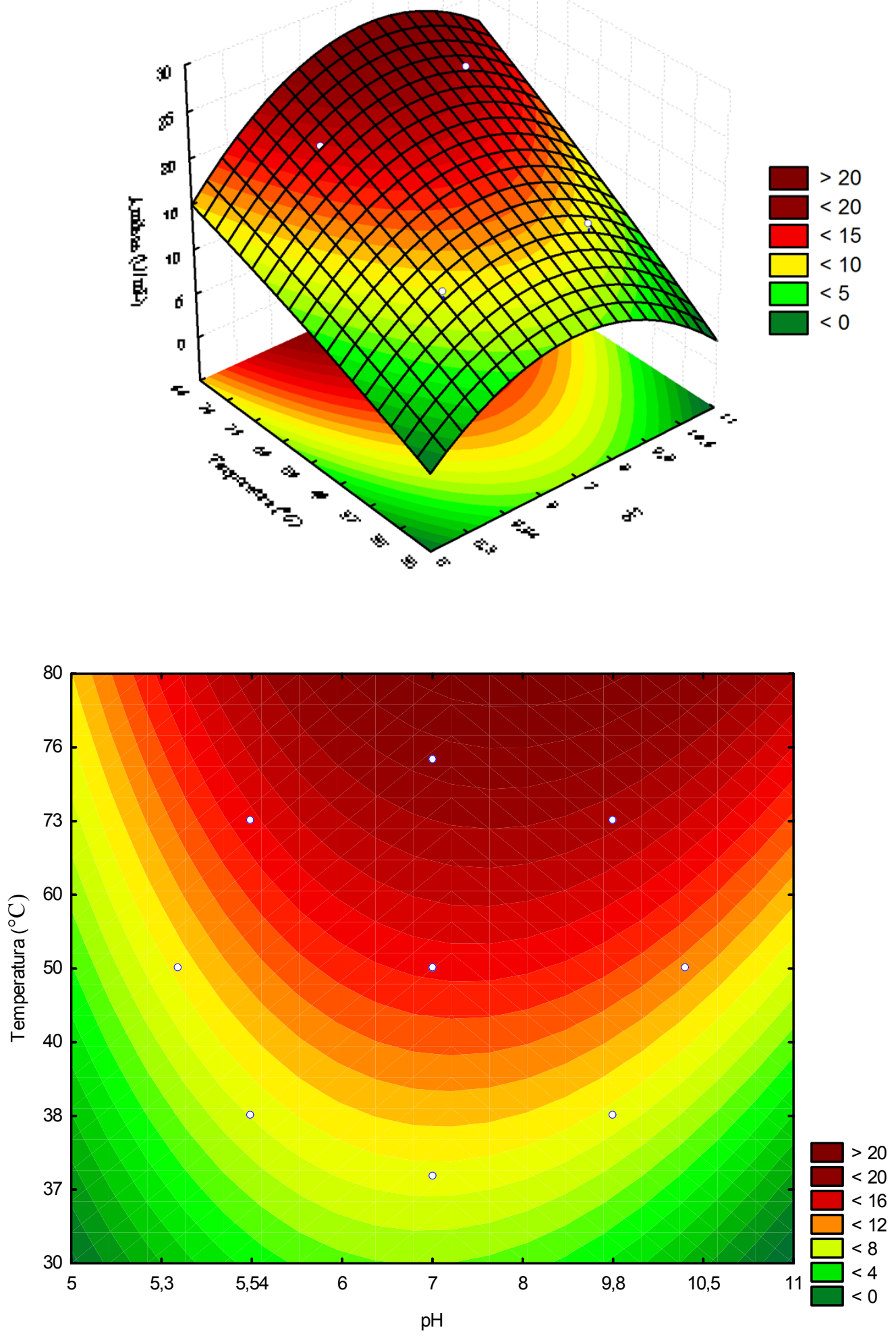


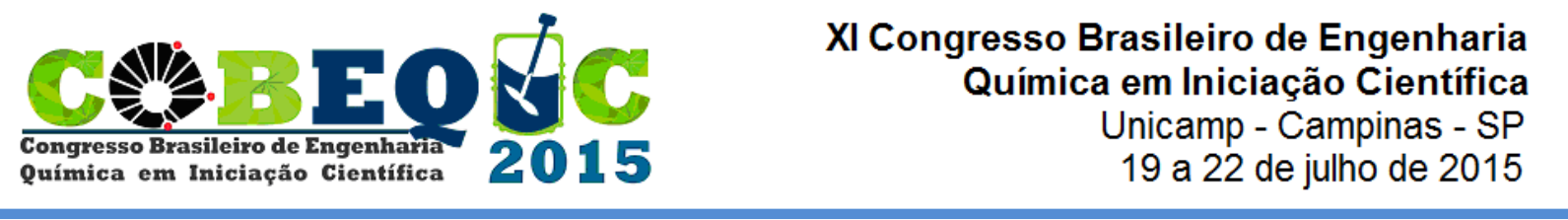

\section{CONCLUSÃO}

$\mathrm{Na}$ caracterização parcial em termos de $\mathrm{pH}$ e temperatura ótimos para a enzima $\alpha$ amilase o maior valor de atividade foi de $8,7 \mathrm{U} / \mathrm{mL}$, com temperatura acima de $60^{\circ} \mathrm{C}$ e $\mathrm{pH}$ na faixa de 7 a 9,8 .

\section{AGRADECIMENTOS} financeiro.

Os autores agradecem a Uri Erechim, FAPERGS, CAPES e CNPq pelo apoio

\section{REFERENCIAS}

FIGUEIRA, E. L. Z.; SÁ, M. C.; IDA, E. I. e HIROOKA, E. Y. Produção e caracterização de amilases de Fusarium moniliforme e Aspergillus flavus. Boletim do Centro de Pesquisa de Processamento de Alimentos, Curitiba, 18, 13-26, 2000.

MILLER, G. L. Use of dinitrosalicyclic acid reagent for determination of reducing sugar. Analytical Chemistry, 31, 426 - 428, 1959.

NELSON, D. L.; COX, M. M. Princípios de bioquímica de Lehninger, 5 Ed. Porto Alegre: Artmed, 2011.

RASIAH, I. A.; REHM, B. H. A. One-step production of immobilized $\alpha$-amylase in recombinant Escherichia coli. Applied and Environmental Microbiology, Washington, 75, 2012 - 2016, 2009.

SANKALIA, M. G.; MASHRU, R. C.; SANKALIA, J. M., et al. Reversed chitosan-alginate polyelectrolyte complex for stability improvement of alpha-amylase: Optimization and physicochemical characterization. European Journal of Pharmaceutics and Biopharmaceutics, 65, 215-232, 2007.

SAN-LANG WANG, YEH-CHEN LIANG, TZU-WEN LIANG. Purification and characterization of a novel alkali-stable _-amylase from Chryseobacterium taeanense TKU001, and application in antioxidant and prebiotic. Process Biochemistry, 46, 745$750,2011$.

SILVA, R. L. F. O. B.; SOUZA, R. R.; SANTANA, J. C. C.; TAMBOURGI, E. B. Imobilização de enzimas de milho maltado em gel. Ciência e Tecnologia de Alimentos, v. 28, n. 3, p. 642-648, 2008.

SPIER, M. R. Produção de enzimas amilolíticas fúngicas $\alpha$-amilase e amiloglucosidase por fermentação no estado sólido. Dissertação de mestrado. Universidade Federal do Paraná. Curitiba - PR, 2005. 143p.

VIEIRA-JÚNIOR, A. Alfa e beta-amilase no metabolismo do amido durante o amadurecimento da banana: clonagem e caracterização molecular. Tese de doutorado. Universidade de São Paulo. São Paulo-SP, 2006. 91p. 